University of Nebraska - Lincoln

DigitalCommons@University of Nebraska - Lincoln

$11-20-2001$

\title{
Interactions of Ultrashort, Ultrahigh Intensity Laser Pulses with Underdense Plasmas
}

\author{
Xiaofang Wang \\ Shanghai Institute of Optics and Fine Mechanics, Chinese Academy of Sciences, Shanghai, China \\ Wei Yu \\ Shanghai Institute of Optics and Fine Mechanics, Chinese Academy of Sciences, Shanghai, China \\ Sterling Backus \\ JILA, University of Colorado, Boulder, CO \\ Margaret Murnane \\ JILA, University of Colorado, Boulder, CO \\ Henry Kapteyn \\ JILA, University of Colorado, Boulder, CO \\ See next page for additional authors
}

Follow this and additional works at: https://digitalcommons.unl.edu/physicsumstadter

Part of the Physics Commons

Wang, Xiaofang; Yu, Wei; Backus, Sterling; Murnane, Margaret; Kapteyn, Henry; and Umstadter, Donald P., "Interactions of Ultrashort, Ultrahigh Intensity Laser Pulses with Underdense Plasmas" (2001). Donald Umstadter Publications. 58.

https://digitalcommons.unl.edu/physicsumstadter/58

This Article is brought to you for free and open access by the Research Papers in Physics and Astronomy at DigitalCommons@University of Nebraska - Lincoln. It has been accepted for inclusion in Donald Umstadter Publications by an authorized administrator of DigitalCommons@University of Nebraska - Lincoln. 


\section{Authors}

Xiaofang Wang, Wei Yu, Sterling Backus, Margaret Murnane, Henry Kapteyn, and Donald P. Umstadter 


\title{
Interactions of Ultrashort, Ultrahigh Intensity Laser Pulses with Underdense Plasmas
}

\author{
Xiaofang Wang', Wei $\mathrm{Yu}^{1}$, Sterling Backus ${ }^{3}$, Margaret Murnane ${ }^{3}$, Henry Kapteyn ${ }^{3}$, and Donald \\ Umstadter ${ }^{2}$ \\ 'Shanghai Institute of Optics and Fine Mechanics, Chinese Academy of Sciences, P.O. Box \\ 800-211, Shanghai 201800, China. \\ ${ }^{2}$ Center for Ultrafast Optical Science, University of Michigan, Ann Arbor, MI 48109, USA. \\ ${ }^{3}$ JILA, University of Colorado, Boulder, CO 80309, USA. \\ Phone: $+86-21-59534890$ \\ Fax: +86-21-59528812 \\ E-mail: wangxf@mail.shcnc.ac.cn
}

\begin{abstract}
The interactions of ultraintense laser pulses with underdense plasmas are studied in a new regime in which the longitudinal spatial extent of the pulse duration is close to both the laser focal spot size and the plasma wavelength.
\end{abstract}

The interaction of ultrashort, high-intensity laser pulses with underdense plasmas is of fundamental interest, relevant to relativistic optics, laser and plasma based accelerators and radiation sources, and ICF fast ignition scheme. However, most previous studies used laser pulse durations with their longitudinal spatial extent much greater than the focal spot size or the plasma wavelength. We will present results in a new regime, in which the pulse's longitudinal spatial extent is close to, or shorter than, both the focal spot size and the plasma wavelength. The results include the generation of a resonant, longitudinal laser wakefield, the observation of relativistic filamentation, and the emission of megaelectronvolt (MeV) electrons which are strongly correlated to the relativistic filamentation.

The experiments were performed with a multi-terawatt, ultrashort Ti:sapphire laser system that delivered $10 \mathrm{~Hz}$ pulses at $810 \mathrm{~nm}$ [2]. The pulse shape and beam quality of the output beam were well characterized. Usually, the pulse duration was $29 \mathrm{fs}$ in full width of maximum (FWHM) with $93 \%$ laser energy contained from \pm 38 fs to the peak. At \pm 200 fs from the peak, the intensity contrast was $10^{-5}$. Under optimized condition, the pulse duration could be as short as $24 \mathrm{fs}$. With an f/4.5, 45-degree off-axis metallic parabola, the 4-cm-diamter beam was focused into a spot of $r_{0}=8 \mu \mathrm{m}$, containing $50 \%$ laser energy inside the first minimum. A supersonic gas jet was used to produce a flat-top-profile gas plume, which was found necessary for the high-intensity laser interactions with underdense plasmas.

A physics picture is introduced to unify the two concepts, relativistic self-focusing and relativistic filamentation. The theoretically predicted filament size is given by $4 \pi c / \omega_{p} a_{0}$ [1]. If the laser focal spot size is $r_{0}$, the necessary condition for relativistic filamentation is given by $4 \pi c / \omega_{p} a_{0} \tilde{<} 2 r_{0}$, where “ $\sim$ " applies to relativistic selffocusing. Note that relativistic self-focusing is the special case of relativistic filamentation.

The evolution from relativistic selffocusing to relativistic filamentation is observed from energy transmission measurement and optical microscopic imaging [3]. It is found that with increasing laser power, the laser energy transmission loss becomes larger. An inflection point of the transmission loss occurs at incident laser power $P \cdot P_{c}$, where $P_{c}$ is the power for relativistic self-focusing. When $P \sim 5 P_{c}$, the 
transmission loss becomes apparent. The apparent transmission loss is verified and caused by the growth of relativistic filamentation, which scatters the laser beam out of its vacuum propagation angle.

Along with the growth of relativistic filamentation, $\mathrm{MeV}$ electrons are observed. The electrons are emitted as a collimated beam in the forward direction. It is interesting to note that, possibly due to the filamentation, the electron beam has a divergence angle of only $1^{\circ}$ in FWHM, the narrowest ever observed from a laser-plasma generated $\mathrm{MeV}$-electron beam. A mechanism has recently been proposed for electron acceleration by a propagating short ultraintense laser pulse [4], which may be the physical cause for the strong correlation of the $\mathrm{MeV}$ electron emission to the filamentation process.

An important issue of the interaction studies is the generation of a largeamplitude, resonant laser wakefield driven by the ultrashort pulse. Different from previous wakefield results that the transverse wakefield was dominant, the onedimensional resonant wakefield is explored in this work. The latter means that the longitudinal wakefield is dominant. Experimental results indicate density resonance, which is consistent with theoretical predictions for a onedimensional resonant laser wakefield. The wakefield amplitude and the duration are measured. Some new features are observed. The details will be presented.

1. C. Max et al., Phys. Rev. Lett. 33, 209 (1974).

2. H. Wang et al., J. Opt. Soc. Am. B16, 1790 (1999).

3. X. Wang et al., Phys. Rev. Lett. 84, 5324 (2000).

4. W. Yu et al., Phys. Rev. Lett. 85, 570 $(2000)$. 